\section{Light my firefly}

Fireflies emit light to attract mates or prey. As Ki-Hun Jeong and colleagues
These

asymmetric

and hierarchical

structures

contribute

substantially

to the efficient

extraction of

bioluminescent

light report in Nano Letters, hierarchical structures on the insect's body are important for the enhancement of the emitted light and can be mimicked in the bioinspired design of an organic light-emitting diode (OLED) to significantly improve its efficiency.

The lantern, the organ that fireflies use to generate light, comprises a dorsal layer, a photogenic layer in which light emission takes place, and a cuticle - the focus of the study. Microscopy images reveal that the cuticle has a complex hierarchical structure composed of asymmetric, inclined microstructures with a nanostructured pattern. As Jeong explains, "our large-scale photonic calculations clearly reveal that these asymmetric and hierarchical structures contribute substantially to the efficient extraction of bioluminescent light, which would otherwise be trapped in the lantern, and wide-angle illumination". The hierarchical structures on the cuticle increase the amount of light that is extracted from the photogenic layer by reducing the total internal reflection - a phenomenon occurring above a critical incidence angle when the light moves from a dense medium to a less dense one - which results in the entrapment of the light. Because the photogenic layer emits light in all directions, the dorsal layer also has an important role, as it reflects the light travelling in the inward direction such that it can be emitted through the cuticle.

The researchers applied this intelligent design to an OLED panel, which included an aluminium layer that represented the dorsal reflecting layer, an organic light-emitting layer (the counterpart of the photogenic layer), and an optical resin layer with asymmetric microstructures and hierarchical structures inspired by the firefly's cuticle. This photonic bioinspired structure can efficiently extract the light confined in the OLED by total internal reflection, leading to an increase in the external quantum efficiency of up to $60 \%$ and to an illumination angle $16 \%$ wider compared with conventional OLEDs. "Our technological breakthrough is the large-scale fabrication of inclined microstructures and of highly ordered nanostructures on each inclined microstructure," says Jeong.
Natural photonic structures, such as compound eyes or butterfly wings, have been mimicked in imaging and display applications, but bioinspired lighting applications still have to be fully explored.

"We strongly believe that biologically inspired OLEDs will set a new paradigm for biomimetic engineering for lighting applications. These results will have a significant impact on the multidisciplinary communities mining smartness from nature," concludes Jeong.

Giulia Pacchioni

ORIGINAL ARTICLE Kim, J. J. et al. Biologically inspired organic light-emitting diodes. Nano Lett. http://dx.doi.org/10.1021/acs.nanolett.5b05183 (2016)

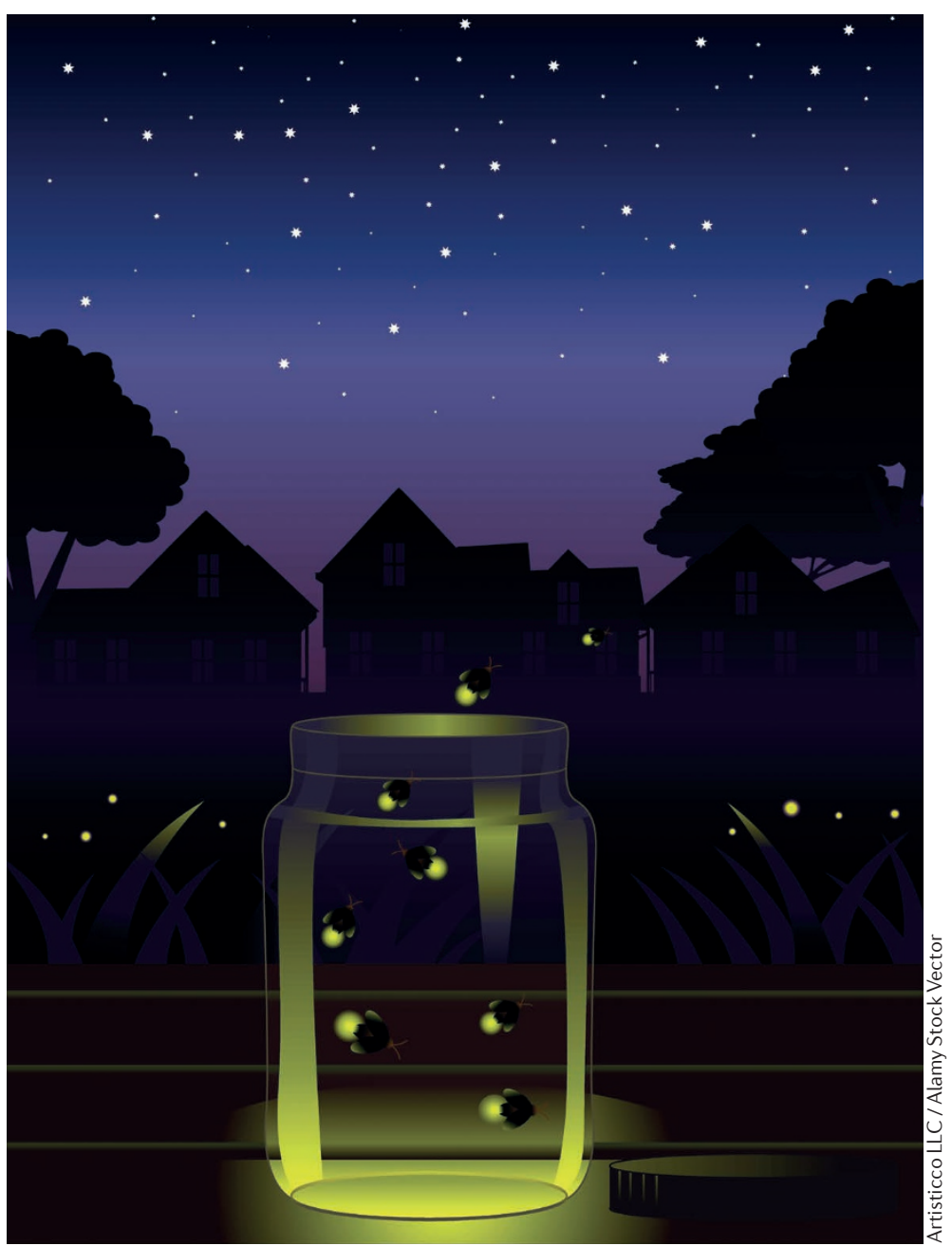

